\title{
Micromechanics Modeling of Functionally Graded Materials Containing Multiple Heterogeneities
}

\author{
Jaesang Yu*广, Cheol-Min Yang**, Yong Chae Jung**
}

\begin{abstract}
Functionally graded materials graded continuously and discretely, and are modeled using modified MoriTanaka and self-consistent methods. The proposed micromechanics model accounts for multi-phase heterogeneity and arbitrary number of layers. The influence of geometries and distinct elastic material properties of each constituent and voids on the effective elastic properties of FGM is investigated. Numerical examples of different functionally graded materials are presented. The predicted elastic properties obtained from the current model agree well with experimental results from the literature.
\end{abstract}

Key Words: functionally graded material (FGM), micromechanics, heterogeneity, composites

\section{INTRODUCTION}

Due to their unique structure and multi-functionality, functionally graded materials (FGMs) are gaining attention and are being proposed for many applications [1-3]. In FGMs, the variation of material properties along a specific direction helps the material to have the desirable material properties of each constituent at a specific material point with structural continuity. For example, ceramic/metal FGMs have the important material properties of metal, such as fracture toughness and high thermal conductivity, without losing the important properties of a ceramic, such as hardness and thermal protection [4-7]. A detailed review on the performance of FGM can be seen in the literature.

To accurately model FGM, knowing the effective or bulk material properties as a function of individual material properties and geometry especially at micromechanics level is essential. In the last few years, different models have been proposed to estimate the effective properties of FGMs with respect to reinforcement volume fractions. Weng [8], investigated the effective bulk moduli of two functionally graded composites by means of change of the dependent variable. Rahman and Chakraborty [9] proposed a stochastic micromechanical model for predicting probabilistic characteristics of three phase FGMs. Pindera et al. [10] used a computational micromechanical model, the generalized method of cells, to predict local stress in the fiber and matrix phases of FGMs. Fang et al. [11], developed a micromechanics-based elastodynamic model to predict the dynamic behavior of two-phase functionally graded materials.

Zuiker [12] reviewed the micromechanical modeling of FGMs and concluded that the self-consistent method (SCM) provided good estimates, with minimal effort, and with no need for empirical fitting of parameters for the silicon carbide (SiC) - carbon (C) FGMs. Gasik [13] studied the efficiency of the simplest micromechanical models to provide the most accurate estimates of FGM components with an arbitrary nonlinear three-dimensionally orientation of phases. Reiter and Dvorak [14] used the transition function with Mori-Tanaka method (MTM) and SCM to predict the thermo-mechanical properties in C/SiC FGMs. Yin et al., [15,16] used MTM, with the effect of particle distance interactions, to predict the elastic and thermoelastic properties of $\mathrm{TiC} / \mathrm{Ni}_{3} \mathrm{Al}$ and $\mathrm{Mo} / \mathrm{SiO}_{2}$ FGMs. Similar models are available for two phase functionally graded materials that contain two different material systems. The actual functionally graded materials can have more than two compositions or constituents, and a model that accounts for multiple heterogeneities is essential. 
In the present study a modified micromechanics model is proposed and extended to estimate the effective elastic properties of FGMs containing multiple heterogeneities with an arbitrary number of coating layers. Different numerical examples are considered and compared with experimental results from the literature.

\section{PROBLEM FORMULATION}

\subsection{Functionally graded materials (FGMs)}

Functionally graded materials can be idealized as a combination of a number of discreet layers of nano-composite, where each of the layers has a defined microstructure that is a function of the volume fraction of the constituents of the material. From a micromechanics modeling point of view, these discrete graded layers can be categorized in three distinct zones.

Let us consider a functionally graded material, where material $\mathrm{A}$, (A could be more than one material) with different geometry (short fibers, platelets, spheres, etc) is reinforced in a base material $\mathrm{B}$, and the volume fraction of $\mathrm{A}$ is increasing from one end to the other end as shown in Fig. 1. As shown in the figure, zone 1 is the region where the reinforcement, phase $A$, volume fraction is lower than the base material, phase $B$, referred to as the phase $\mathrm{B}$ dominant zone. Zone 3 is the region where the volume fraction of the reinforcement, phase $A$, is higher than the base material, phase $\mathrm{B}$, referred to as the phase A dominant zone. Zone 2 is the region where the volume fraction of phase A and phase B cannot be clearly defined, referred to as the transition zone. Each layer in Zone 1 and Zone 3 can, in principle, be modeled similarly using a micromechanics approach as the volume fraction between the two phases are distinct enough to satisfy the main assumption.

The main challenge is dealing with the transition zone, where the matrix and the reinforcement are not sufficiently distinguishable. Recently, a different method has been proposed to overcome this problem and model the transition

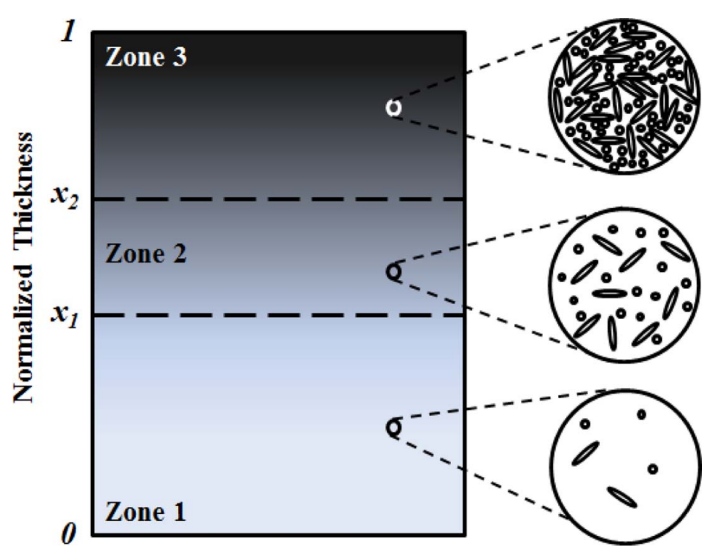

Fig. 1. Schematic illustration of multi-phase functionally graded materials (FGMs) zone. In this zone a linear phenomenological transition function introduced by Reiter and Dvorak [14] is adopted.

\subsection{Micromechanics modeling}

2.2.1 Modified Mori-Tanaka method (MTM) for FGM with multiple heterogeneities and multiple layers

In MTM, the effective material properties for composites containing arbitrary ellipsoidal heterogeneities can be obtained using the continuum-averaged stress and strain fields [17-20]. For two phase composites, containing a matrix phase (0) and a particle phase (1), the effective 4 th order elastic stiffness tensor, $\mathrm{L}$, can be expressed as

$$
\begin{gathered}
\bar{L}=L^{(0)}:\left\{I+c_{1}\left(S^{(1)}-I\right):\left(A^{(1)}-S^{(1)}\right)^{-1}\right\}: \\
\left\{I+c_{1} S^{(1)}:\left(A^{(1)}-S^{(1)}\right)^{-1}\right\}^{-1}
\end{gathered}
$$

where

$$
A^{(1)}=\left(L^{(0)}-L^{(1)}\right)^{-1}: L^{(0)}
$$

is the local strain concentration tensor for the particles, $L(0)$ and $L(1)$ are the 4 th rank elastic stiffness tensors for the matrix and particles, $\mathrm{cl}$ is the particles volume fraction, $S(1)$ is the 4 th rank Eshelby [19-21] tensor for the particles, and I is the 4th rank identity tensor. Here a colon ":" is used to denote the tensor double dot product. The Eshelby tensor $(S(1))$ accounts for the influence of the aspect ratio/geometry of the particles on the local strain field. Eshelby tensors for specific reinforcement shapes (spheres, platelets, fibers, etc.) are readily available in the literature $[19,20]$. For FGMs, the effective elastic property for each layer through the thickness direction can be estimated by employing Eq. (5) with either normalized thickness $0 \leq \mathrm{x} \leq$ 1 or volume fractions of phase (0) and phase (1).

Consider a composite with $\mathrm{m}$ types of distinct ellipsoidal heterogeneities $(\mathrm{k}=1,2, \ldots, \mathrm{m})$ in the matrix $(0)$, where each heterogeneities consists of $n$ layers $(\alpha k=1,2, \ldots, n k)$ graded in the direction of thickness, having a normalized thickness $0 \leq \mathrm{z}$ $\leq 1$. Each heterogeneity has a distinct elastic property, shape, and orientation distribution. The strain and stress fields at any location $\mathrm{z}$ with heterogeneities, with arbitrary number of layers, can be given by

$$
\begin{aligned}
& \langle\varepsilon\rangle_{k}^{\alpha_{k}}=\varepsilon^{\infty}+S_{(k)}: \varepsilon^{{ }^{*} \alpha^{\alpha_{k}}} \\
& \langle\sigma\rangle_{k}^{\alpha_{k}}=L^{(0)}:\left\{\varepsilon^{\infty}+\left(S_{(k)}-I\right): \varepsilon^{{ }_{k} \alpha_{k}}\right\}
\end{aligned}
$$

where the homogenizing eigenstrain $\left(\varepsilon^{{ }^{*}{ } \alpha_{k}}\right)$ is

$$
\varepsilon^{{ }^{*} \alpha_{k}}=\left(A_{(k)}^{\left(\alpha_{k}\right)}-S_{(k)}\right)^{-1}: \varepsilon^{\infty}
$$

where is the local strain concentration tensor for the $\alpha \mathrm{k}$ th layer of the kth heterogeneity $(\alpha \mathrm{k}=1,2, \ldots, \mathrm{nk}, \mathrm{k}=1,2, \ldots, \mathrm{m})$ 
of each FGM layer through the thickness direction and can be given as

$$
A_{(k)}^{\left(\alpha_{k}\right)}=\left(L^{(0)}-L_{(k)}^{\left(\alpha_{k}\right)}\right)^{-1}: L^{(0)}
$$

Here is the 4th rank elastic stiffness tensor for the $\alpha \mathrm{k}$ th layer of the kth heterogeneity for FGM layers through the thickness direction. By substituting Eqs. (5-6) into Eqs. (3-4), the average strain and stress fields over the infinite domain can be obtained as

$$
\begin{aligned}
& \langle\varepsilon\rangle_{D}=\varepsilon^{\infty}+\sum_{k=1}^{m}\left[\sum_{\alpha_{k}=1}^{n_{k}} c_{(k) \alpha_{k}} S_{(k)}:\left(A_{(k)}^{\left(\alpha_{k}\right)}-S_{(k)}\right)^{-1}\right]: \varepsilon^{\infty} \\
& \langle\sigma\rangle_{D}=L^{(0)}:\left\{\varepsilon^{\infty}+\sum_{k=1}^{m}\left[\sum_{\alpha_{k}=1}^{n_{k}} c_{(k) \alpha_{k}}\left(S_{(k)}-I\right):\left(A_{(k)}^{\left(\alpha_{k}\right)}-S_{(k)}\right)^{-1}\right]: \varepsilon^{\infty}\right\}
\end{aligned}
$$

The overall elasticity tensor, $\bar{L}$, for a composite containing $\mathrm{m}$ distinct types of heterogeneities $(\mathrm{k}=1,2, \ldots, \mathrm{m})$ with each having an arbitrary number of layers (nk), in a matrix phase (0) can be expressed as

$$
\begin{gathered}
\bar{L}=L^{(0)}:\left\{I+\sum_{k=1}^{m}\left[\sum_{\alpha_{k}=1}^{n_{k}} c_{(k) \alpha_{k}}\left(S_{(k)}-I\right):\left(A_{(k)}^{\left(\alpha_{k}\right)}-S_{(k)}\right)^{-1}\right]\right\} \\
\left\{I+\sum_{k=1}^{m}\left[\sum_{\alpha_{k}=1}^{n_{k}} c_{(k) \alpha_{k}} S_{(k)}:\left(A_{(k)}^{\left(\alpha_{k}\right)}-S_{(k)}\right)^{-1}\right]\right\}^{-1}
\end{gathered}
$$

Here $c_{(k) \alpha_{k}}$ is the volume fraction of the $\alpha \mathrm{k}$ th layer of the kth heterogeneity, and $\bar{L}$ and $L^{(0)}$ are the effective elastic property, and matrix phase elastic stiffness in each FGM layer through the normalized thickness direction, $0 \leq \mathrm{x} \leq 1$. Unlike in the case of two phase formulation given by Eq. (1), overall elasticity tensor, $\bar{L}$, given by Eq. (9) is a function of multiple material inhomogenity, $c(k)$, and number of layers, $\alpha \mathrm{k}$. Once the overall elasticity tensor for each layer of FGMs, $\bar{L}$, is determined, the relevant elastic moduli can be determined using an orientation averaging scheme. A detailed discussion along with computational procedures of an orientation averaging scheme is available in the paper by Yu et al. [22-24].

2.2.2 Modified self-consistent method (SCM) for FGM with multiple heterogeneities and multiple layers

Generally, when using the Self-Consistent Method, it is assumed that a single ellipsoidal heterogeneity is embedded within a homogeneous matrix with an unknown effective stiffness tensor [22-24]. It is easy to prove that the effective elastic tensor given by the SCM for FGM layers through the nor- malized thickness direction, $0 \leq \mathrm{x} \leq 1$, can be obtained from the MTM model by setting . Unlike MTM, however, in the SCM model the effective elastic tensor of the homogenized matrix is unknown, and an iterative procedure is required. In this model, first an initial effective elastic tensor will be assumed, and with an iteration scheme the final tensor will be determined. Suppose that the effective homogeneous medium with elastic stiffness tensor, $\bar{L}$, contains $\mathrm{m}$ distinct types of ellipsoidal heterogeneities $(\mathrm{k}=1,2, \ldots, \mathrm{m})$, each consisting of nk layers $(\alpha \mathrm{k}=1,2, \ldots, \mathrm{nk})$ in a matrix phase $(0)$. Then the overall elasticity tensor, $\bar{L}$, the complete formulation is given in the literature [22-24], can be expressed as

$$
\bar{L}=f\left(\bar{L}, S_{(k)}, A_{(k)}^{\left(\alpha_{k}\right)}, c_{(k) \alpha_{k}}\right)
$$

For the case of aligned, two-dimensional randomly oriented and three-dimensional randomly oriented heterogeneities, the calculated elasticity tensor for each layer of FGMs will display orthotropic, transversely isotropic, and isotropic material symmetries, respectively. In the present study, the elastic moduli for each layer of FGMs containing three-dimensionally oriented heterogeneities will be calculated.

\section{EFFECTIVE ELASTIC MODULI FOR FGMS CONTAINING MULTIPLE HETERO- GENEITIES}

\subsection{Effect of material properties and geometry of each constituent}

The modified micromechanics model developed and discussed above is implemented for FGMs with discrete gradation, where each of the layers in the gradation is considered as a composite containing multiple heterogeneities. In all numerical examples of FGMs, such as silica spheres, hollow cenospheres, and voids considered, all heterogeneities are assumed to be well dispersed in the base material and perfectly bonded to the other phases. Table 1 contains a summary of relevant mechanical properties of materials used in the calculation. In

\begin{tabular}{|c|c|c|}
\hline $\begin{array}{l}\text { Reinforcement/ } \\
\text { Matrix }\end{array}$ & $\begin{array}{l}\text { Reinforcement } \\
\text { dimensions }\end{array}$ & $\begin{array}{l}\text { Young's modulus } \\
\text { (GPa) }\end{array}$ \\
\hline \multirow{2}{*}{$\begin{array}{l}\text { Hollow cenosphere/ } \\
\text { Polyester }\end{array}$} & \multirow{2}{*}{$\begin{array}{c}\text { Ds }=127 \mu \mathrm{m} \\
\text { tw }=12.7 \mu \mathrm{m} \\
\text { (Parameswaran and } \\
\text { Shukla, }[25])\end{array}$} & $\begin{array}{c}\text { Es }=175.0 \\
(\text { Parameswaran and } \\
\text { Shukla, }[25]) \\
\end{array}$ \\
\hline & & $\begin{array}{c}\mathrm{Em}=3.5 \\
\text { (Parameswaran and } \\
\text { Shukla, }[25])\end{array}$ \\
\hline
\end{tabular}
all calculations, the FGMs' elastic properties were normalized by the base material elastic property, E1, in order to clearly illustrate the effect of the heterogeneities.

Table 1. Selective material properties and geometries of FGM considered 


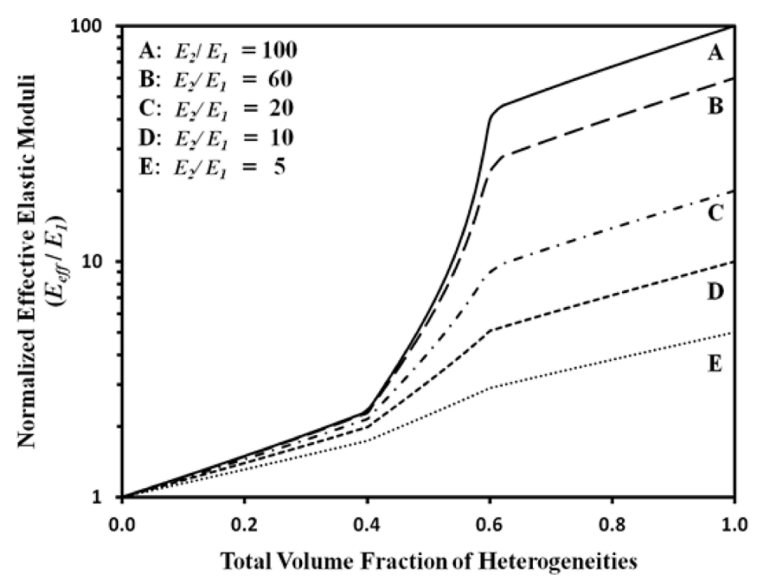

Fig. 2. Effect of elastic properties of individual materials (E2/E1) on predicted effective elastic moduli of FGMs

\subsubsection{Effect of individual material properties}

To understand the effect of the individual elastic properties on the effective elastic properties of the FGM, different ratios of elastic material properties were considered. Fig. 2 shows a plot of the predicted normalized effective elastic moduli (Eeff / E1) of FGM, for different E2/E1 ratio, as a function of the volume fraction of the reinforcement. The effective elastic moduli of FGM for all E2/E1 ratios are increased as the volume fraction of reinforcement increases, and linearly in the case of zone 1 and zone 3. For a relatively low volume fraction of heterogeneity (phase 1 , cs $\leq 0.4$ ), the difference in elastic properties between the heterogeneity and the base material does not affect the effective elastic properties very much. For a higher volume fraction of the heterogeneity (phase 3, $0.6 \leq \mathrm{cs}$ ), however, the effective elastic properties increases as the ratio between the two material elastic properties increases. Furthermore, there is a jump in effective elastic properties and a trained change from zone 1 to zone 2 and from zone 2 to zone 3. The jump in effective elastic moduli on the transition zone is significant for material with a large difference in elastic material property $(\mathrm{E} 2 / \mathrm{E} 1>>1)$. Thus, the effective elastic moduli strongly depend on volume fraction and material properties of each constituent.

\subsubsection{Effect of reinforcement geometry}

To investigate the effect of reinforcement geometries on the effective elastic moduli of FGMs, different geometries of reinforcements such as a) fibers, b) platelets, and c) spheres are considered. In this calculation, the ratio of elastic properties between the reinforcement and the base material is set to be constant at E2/E1 = 20. The lower and upper boundaries of the volume fractions of the reinforcement for zone 2 were set $40 \%$ and $60 \%$ respectively. Aspect ratios, $\mathrm{AR}=100$, for fibers (length/diameter) and platelets (diameter/thickness) were used. As shown in Fig. 3, the elastic moduli for FGM containing randomly oriented fibers were higher than those pro-

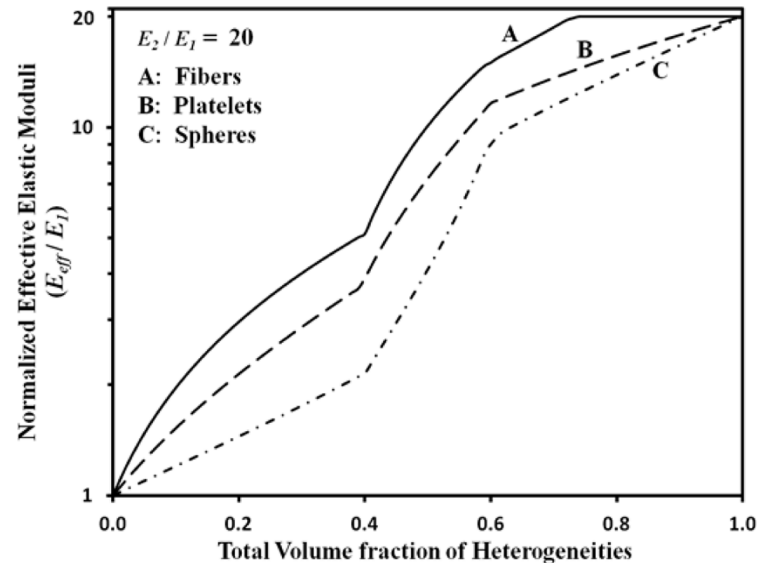

Fig. 3. Effect of geometry (fibers, platelets, spheres) of individual materials on predicted effective elastic moduli of FGMs

duced by the spheres or the platelets for the same volume contents of reinforcements. It must be noted that the micromechanics model was not able to predict the effective elastic moduli for FGM containing fibers in the range of high volume fraction $(0.75 \leq \mathrm{cf})$ due to a violation of the basic assumption of micromechanics.

\subsection{Numerical examples}

3.2.1 Linearly varying FGM made of matrix reinforced with hollow cenospheres

Parameswaran and Shukla [25] fabricated FGM consisting of polyester matrix and hollow cenospheres with $=127 \mu \mathrm{m}$ (Ds) diameter and $12.7 \mu \mathrm{m}$ wall thickness (tw). The reinforcements have a thin-walled "egg-shell" type of architecture. The wall-thickness to diameter ratio, tw/Ds is about 0.1. Fig. 4 shows an optical micrograph of hollow cenospheres in a polyester matrix and experimentally measured normalized elastic moduli as a function of volume fraction of the cenospheres

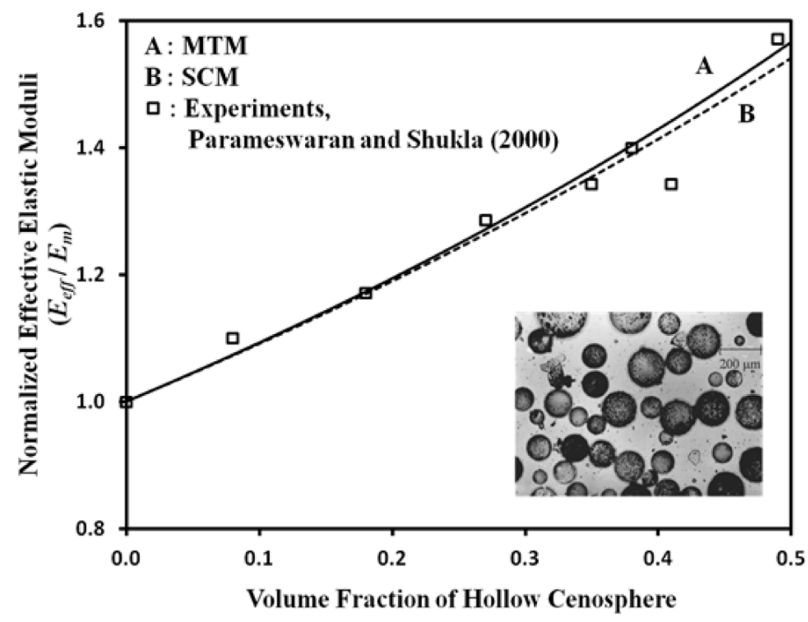

Fig. 4. Measured [25] and predicted effective elastic moduli for hollow cenosphere/polyester FGM (Image from [25]) 


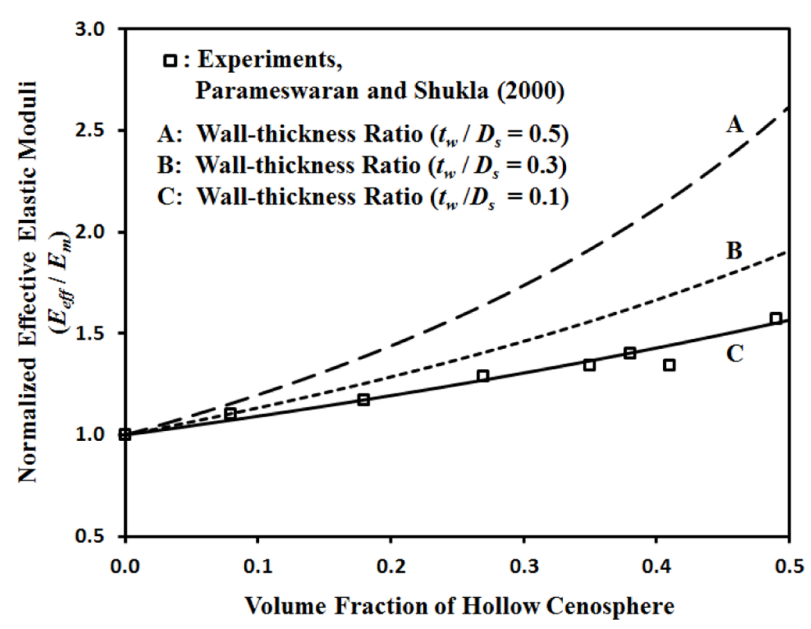

Fig. 5. Effect of cenosphere wall-thickness ratio (tw/Ds) on predicted effective elastic moduli for hollow cenosphere/ polyester FGM

[25]. The tensile moduli increase with increase in hollow cenospheres volume fraction as shown in Fig. 4 . This suggests that the very thin silica outer wall contributes to the effective FGM properties. In our micromechanics model, the hollow cenospheres were simulated using a two-layer system, where the inner layer was ascribed essentially as voids with null material properties and the outer layer considered as a solid layer of silica. Fig. 4 shows the effective elastic moduli of the FGM, both experimental and numerical, as a function of the hollow cenospheres volume fraction. The solution from the current micromechanical model reasonably agrees with the experimentally measured value.

On the other hand, effective elastic properties were predicted for FGM with cenospheres having different wall-thickness to diameter ratios (tw/Ds) in a polyester matrix as shown in Fig. 5. Three distinct wall-thicknesses to diameter ratios, tw/ $\mathrm{Ds} \approx 0.5,0.3$, and 0.1 , were considered for comparison. The effective elastic modulus for FGM that contains cenospheres with thick wall-thickness to diameter ratio ( $t w / D s \approx 0.5)$ was higher than that of FGM containing reinforcement with thin wall-thickness to diameter ratio (tw/Ds $\approx 0.1$ ). Again, this supports the argument that, the reinforcement outer wall thickness significantly contributes to the overall FGM properties.

3.2.2 FGM composed of titanium monoboride (TiB) and titanium (Ti) materials

Hill et al. [4] investigated and measured elastic properties for a FGM composed of ceramic (titanium monoboride (TiB2)) and metal (titanium (Ti)). Fig. 6 shows a plot of an optical micrograph of TiB/Ti FGM and experimentally measured normalized elastic moduli as a function of volume fraction of the TiB [4]. As can be seen in the figure, experimentally measured effective stiffness for the FGM increases with

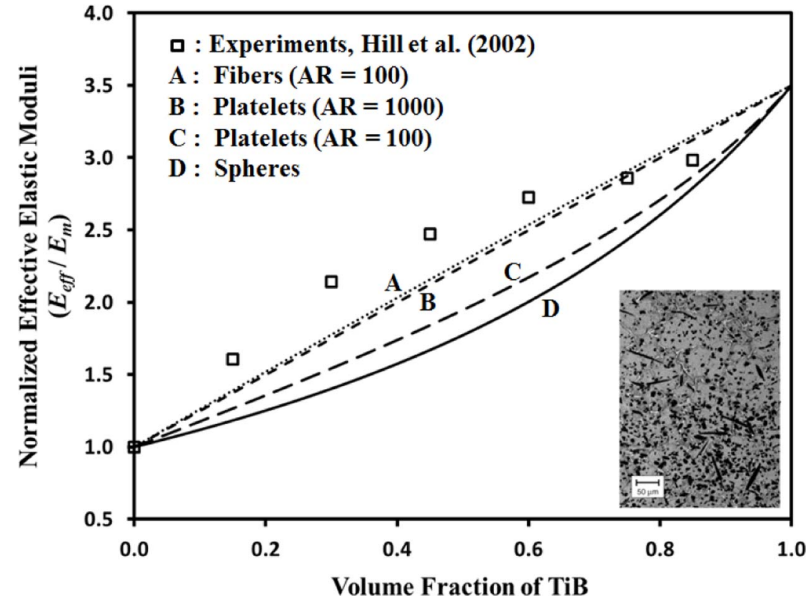

Fig. 6. Measured [4] and predicted effective elastic moduli for TiB/Ti FGM (Image from [4])

increase in volume fraction of TiB. In the current model, the metal was considered as a base material and the ceramic as reinforcement. It should be mentioned that, as shown in the microscopic image in Fig. 6, TiB appears in different shapes, such as spheres, fibers etc. In this case the FGM was modeled with metal (Ti) as the base material, reinforced with different shapes (i.e., fibers, platelets, and spheres) of ceramic (TiB). The aspect ratio (length to diameter ratio, AR) for fibers was 100 , and for the platelets were 100 and 1000 . Here only shows the case for composites containing a single heterogeneity problem such as fibers, platelets, and spheres rather than multiple heterogeneities problems (combination of heterogeneities in matrix) due to the fact that curve A (fibers, AR = 100) almost reached the upper bound (Voigt) of composites in elastic modulus itself. On the other hands, Curve D (spheres) also reached the lower bound (Reuss) of composites. Thus, the results for the case of combination of fibers and platelets (or spheres) obtained from micromechanics models should lower than the upper bound (fibers only). Even though the effective FGM elastic moduli depends on TiB shapes, in all cases the numerical results were lower than experimentally measured data.

\section{CONCLUSIONS}

The effective elastic properties of functionally graded materials (FGMs) containing multiple distinct heterogeneities are predicted using classical micromechanical model. The effective stiffness of different material has been considered as a numerical example and verified with experimental results from the literature. The numerical examples were in reasonably good agreement with experimental results from the literature. It is found that the effective elastic stiffness is highly dependent on the individual constituent elastic properties and geometries of the reinforcements. 


\section{ACKNOWLEDGEMENTS}

This paper was supported by the Korea Institute of Science and Technology (KIST), Jeonbuk branch agency specific enterprise with the contract number $2 \mathrm{Z} 03870$.

\section{REFERENCES}

1. Miyamoto, Y., Kaysser, W.A., Rabin, B.H., Kawasaki, A., Ford, and R.G., Functionally Graded Materials: Design, Processing and Applications. Kluwer Academic Publishers, First ed., Dordrecht. 1999.

2. Pompea, W., Worch, H., Epple, M., Friess, W., Gelinsky, M., Greil, P., Hempele, U., Scharnweber, D., and Schulte, K., "Functionally Graded Materials for Biomedical Applications," Material Science and Engineering A, Vol. 362, 2003, pp. 40-60.

3. Mller, E., Draar, C., Schilz, J., and Kaysser, W.A., "Functionally Graded Materials for Sensor and Energy Applications," Material Science and Engineering, Vol. 362, 2003, pp. 17-30.

4. Hill, M.R., Carpenter, D., Paulino, G.H., Munir, Z.A., and Gibeling, J.C., "Fracture Testing of a Layered Functionally Graded Material. Fracture Resistance Testing of Monolithic and Composite Brittle Materials," ASTM STP 1409, J.A. Salem, G.D. Quinn, G.D., and M.G. Jenkins. eds., American Society for Testing and Materials, West Conshohocken, PA, 2002.

5. Kidane, A., and Shukla, A., "Dynamic Constitutive Behavior of Ti/TiB FGM under Thermo Mechanical Loading," Journal of Material Science, Vol. 43, 2003, pp. 2771-2777.

6. Kidane, A., and Shukla, A., "Quasi-Static and Dynamic Fracture Initiation Toughness of $\mathrm{Ti} / \mathrm{TiB}$ Layered Functionally Graded Material under Thermo-Mechanical Loading," Engineering Fracture Mechanics, Vol. 77, 2010, pp. 479-491.

7. Birman, V., and Byrd, L.W., "Modeling and Analysis of Functionally Graded Materials and Structures," Applied Mechanics Reviews, Vol. 60, 2007, pp. 195-216.

8. Weng, G.J., "Effective Bulk Moduli of Two Functionally Graded Composites," Acta Mechanica, Vol. 166, 2003, pp. 57-67.

9. Rahman, S., and Chakraborty, A., "A Stochastic Micromechanical Model for Elastic Properties of Functionally Graded Materials" Mechanics of Materials, Vol. 39, 2007, pp. 548-563.

10. Pindera, M., Aboudi, J., and Arnold, S., "Limitations of the Uncoupled, RVE-Based Micromechanical Approach in the Analysis of Functionally Graded Composites," Mechanics of Materials, Vol. 20, 1995, pp. 77-94.

11. NFang, X. Hu, Q.C., and Huang, W.H., "Determination of Dynamic Effective Properties in Functionally Graded Materials," Acta Mechanica, Vol. 192, 2007, pp. 49-63.
12. Zuiker, J., "Functionally Graded Materials: Choice of Micromechanics Model and Limitations in Property Variation," Composite Engineering, Vol. 5, 1995, pp. 807-819.

13. Gasik, M., "Micromechanical Modeling of Functionally Graded Materials," Computational Material Science, Vol. 13, 1998, pp. 42-55.

14. Reiter, T., and Dvorak, G.J., "Micromechanical Models for Graded Composite Materials," Journal of Mechanics and Physics of Solids, Vol. 46, 1997, pp. 1655-1673.

15. Yin, H.M., Sun, L.Z., and Paulino, G.H., "MicromechanicsBased Elastic Model for Functionally Graded Materials with Particle Interactions," Acta Materilia, Vol. 52, 2004, pp. 35353543.

16. Yin, H.M., Paulino, G.H., Buttlar, W.G., and Sun, L.Z., "Micromechanics-Based Thermoelastic Model for Functionally Graded Particulate Materials with Particle Interactions," Journal of Mechanics and Physics of Solids, Vol. 55, 2007, pp. 132-160.

17. Mori, T., and Tanaka, K., "Average Stress in Matrix and Average Elastic Energy of Materials with Misfitting Inclusions," Acta Metallurgica et Materialia, 21, 1973, pp. 571-574.

18. Benveniste, Y., "A New Approach to the Application of MoriTanaka's Theory in Composite Materials," Mechanics of Materials, Vol. 6, 1987, pp. 147-157.

19. Mura, T., "Micromechanics of Defects in Solids," Second ed., Martinus Nijhoff, Dordrecht. 1987.

20. Nemat-Nasser, and Hori, M., Micromechanics: Overall Properties of Heterogeneous Materials. Elsiver, Second ed., NorthHolland, Amsterdam. 1993.

21. Eshelby, J.D., "The Determination of the Elastic Field of an Ellipsoidal Inclusion and Related Problems," Proceedings of the Royal Society A241, 1957, pp. 376-396.

22. Yu, J., Lacy, T.E., Toghiani, H., and Pittman, C.U., "Classical Micromechanics Modeling of Nanoreinforced Composites with Carbon Nanofibers and Interphase," Journal of Composite Materials, Vol. 45, 2011, pp. 2401-2414.

23. Yu, J., Lacy, T.E., Toghiani, H., and Pittman, C.U., "Effective Property Estimates For Composites Containing Multiple NanoHeterogeneities: Part I Nanospheres, Nanoplatelets, and Voids," Journal of Composite Materials, Vol. 47, 2012, pp. 549-558.

24. Yu, J., Lacy, T.E., Toghiani, H., and Pittman, C.U., "Effective Property Estimates for Composites Containing Multiple NanoHeterogeneities: Part II Nanofibers \& Voids," Journal of Composite Materials, Vol. 47, 2012, pp. 1273-1282.

25. Parameswaran, V., and Shukla, A., "Processing and Characterization of A Model Functionally Graded Material," Journal of Material Science, Vol. 35, 2002, pp. 21-29. 